This is a self-archived version of an original article. This version may differ from the original in pagination and typographic details.

Author(s): Koskinen, Pekka; Kit, Oleg

Title: Efficient approach for simulating distorted materials

Year: 2010

Version: Published version

Copyright: @ 2010 The American Physical Society

Rights: In Copyright

Rights url: http://rightsstatements.org/page/lnC/1.0/?language=en

Please cite the original version:

Koskinen, P., \& Kit, O. (2010). Efficient approach for simulating distorted materials. Physical Review Letters, 105, 106401. https://doi.org/10.1103/PhysRevLett.105.106401 


\title{
Efficient Approach for Simulating Distorted Materials
}

\author{
Pekka Koskinen*, ${ }^{* \dagger}$ and Oleg O. Kit \\ NanoScience Center, Department of Physics, University of Jyväskylä, 40014 Jyväskylä, Finland
}

(Received 26 January 2010; published 30 August 2010)

\begin{abstract}
The operation principles of nanoscale devices are based upon both electronic and mechanical properties of materials. Because these properties can be coupled, they need to be investigated simultaneously. At this moment, however, the electronic structure calculations with custom-made long-range mechanical distortions are impossible, or expensive at best. Here we present a unified formalism to solve exactly the electronic structures of nanomaterials with versatile distortions. We illustrate the formalism by investigating twisted armchair graphene nanoribbons with the least possible number of atoms. Apart from enabling versatile material distortions, the formalism is capable of reducing computational costs orders of magnitude in various areas of science and engineering.
\end{abstract}

DOI: 10.1103/PhysRevLett.105.106401

PACS numbers: 71.15.Dx, 62.25. $-\mathrm{g}, 68.65 . \mathrm{Pq}$

Bloch's theorem has been the propulsive force of computational materials research for more than 80 years [1], today as important as ever. While the theorem is still associated with Bravais lattices and translational symmetry, nanoscience has brought us low-dimensional structures, tubes, tori, wires, and membranes, which get twisted, bent, wrapped, and rippled in experiments. Translational symmetry hides deceiving simulation constraints, since materials cannot distort the way they would prefer, and restricts realistic modeling of nanoelectromechanical components.

Distortions are relevant in a number of topical material systems: polymers, double helices like DNA, lipid bilayers, nanoscrolls, nanocoils, nanowires, and, especially, carbon nanostructures including fullerenes, carbon nanotubes (CNTs), graphene, and graphene nanoribbons (GNRs), to mention a few [2]. For example, materials with high aspect ratio such as CNTs and GNRs get bent [3-5] and thin sheets such as graphene get rippled [3,6,7], unless carefully placed on a support. Classical modeling of distortions is a mature subject [8-10], but while classical interaction potentials and finite element methods give materials' mechanical properties, they are useless for electronic properties. In nanoscience quantum-mechanical modeling is preferred.

How can we include quantum mechanics into these distortion simulations? For decades chemists have used group theory and molecular symmetries to reduce computational costs. In computational materials physics, symmetries beyond translation have been used mainly for chiral carbon nanotubes, in work pioneered by White, Robertson, and Mintmire [11], followed by Popov [12] and Dumitrică [13], with co-workers. Nanotubes are natural because chiral symmetry itself suggests "symmetry adaption"; it is, however, less evident to break the symmetry and investigate the elastic properties in a broader sense.

In this Letter we shall present a compact, exact, and flexible formalism to solve the electronic structure of nano- materials with custom-made distortions. By expanding the concepts of periodicity and simulation cells, the formalism can also reduce computational costs, even in classical materials modeling.

The formalism is obtained by revising Bloch's theorem, and the derivation is straightforward. Consider electrons in a potential $V(\boldsymbol{r})$ that remains invariant in symmetry operations $\mathcal{S}^{n}$,

$$
\hat{D}\left(\mathcal{S}^{n}\right) V(\boldsymbol{r})=V\left(\mathcal{S}^{-\boldsymbol{n}} \boldsymbol{r}\right)=V(\boldsymbol{r}) .
$$

The operation $\mathcal{S}^{n}$, with inverse $\mathcal{S}^{-n}$, is a succession of $n_{i}$ times operation $\mathcal{S}_{i}$ for all $i=1,2, \ldots$, that is $\mathcal{S}^{n}=$ $\mathcal{S}_{1}^{n_{1}} \mathcal{S}_{2}^{n_{2}} \cdots$ with $\boldsymbol{n}=\left(n_{1}, n_{2}, \cdots\right)$. $\mathcal{S}_{i}$ can be any symmetry operation, such as translation, rotation, reflection, joined translation + rotation, or joined translation + reflection, to mention five, and they should form an Abelian group.

Let us give a couple of familiar examples: for bulk $\mathcal{S}_{i}$ 's are three translations; for a benzene ring $\left(\mathrm{C}_{6} \mathrm{H}_{6}\right) \mathcal{S}_{1}$ could be a two-, three-, or sixfold rotation around the symmetry axis; for an achiral carbon nanotube $\mathcal{S}_{1}$ could be a translation along the symmetry axis and $\mathcal{S}_{2}$ could be, say, an $M$-fold rotation around the symmetry axis; for polyethylene $\left(\left[-\mathrm{CH}_{2} \mathrm{CH}_{2}-\right]_{n}\right) \mathcal{S}_{1}$ could be a translation across one $\mathrm{CH}_{2}$ unit followed by a reflection (normally $\mathcal{S}_{1}$ would be a translation across the whole $\mathrm{CH}_{2} \mathrm{CH}_{2}$ unit).

Now, returning to the derivation, since the transformations are isometric, the kinetic energy term in the Hamiltonian

$$
\hat{H}=-\frac{\hbar^{2}}{2 m_{e}} \nabla^{2}+V(\boldsymbol{r})
$$

remains invariant, and $\hat{H}$ commutes with $\hat{D}\left(\mathcal{S}^{n}\right)$, the two operators consequently sharing the same eigenstates. We denote these eigenstates $\psi_{a \boldsymbol{\kappa}}(\boldsymbol{r})$, with $\boldsymbol{\kappa}=\left(\kappa_{1}, \kappa_{2}, \ldots\right)$. Hence we have

$$
\hat{D}\left(\mathcal{S}_{1}^{p}\right) \psi_{a \boldsymbol{\kappa}}(\boldsymbol{r})=\lambda\left(\kappa_{1}\right)^{p} \psi_{a \boldsymbol{\kappa}}(\boldsymbol{r}), \quad(p \text { integer })
$$

where $\lambda\left(\kappa_{1}\right)$ is the eigenvalue of $\hat{D}\left(\mathcal{S}_{1}\right)$. Since electron 
density remains invariant under symmetry operations,

$$
\left|\lambda\left(\kappa_{1}\right)^{p} \psi_{a \boldsymbol{\kappa}}(\boldsymbol{r})\right|=\left|\psi_{a \boldsymbol{\kappa}}(\boldsymbol{r})\right|,
$$

we get $\lambda\left(\kappa_{1}\right)=\exp \left[i \alpha\left(\kappa_{1}\right)\right]$. Now we impose periodic boundary conditions by making the group cyclic $\mathcal{S}_{1}^{M_{1}} \equiv$ $\hat{1}$, and get $\alpha\left(\kappa_{1}\right) \equiv \kappa_{1}=2 \pi m_{1} / M_{1}$, with integers $M_{1}$ and $m_{1} \in\left[0, M_{1}-1\right]$.

By repeating the above steps for the remaining symmetry operations, we obtain a revised Bloch's theorem: in a potential, that is invariant in symmetry operations $\mathcal{S}^{n}$, the energy eigenstates $\psi_{a \boldsymbol{\kappa}}$ at $\boldsymbol{r}$ and at $\boldsymbol{r}^{\prime}=\mathcal{S}^{-\boldsymbol{n}} \boldsymbol{r}$ differ by a phase factor $\exp (i \boldsymbol{\kappa} \cdot \boldsymbol{n})$,

$$
\hat{D}\left(\mathcal{S}^{n}\right) \psi_{a \boldsymbol{\kappa}}(\boldsymbol{r})=\psi_{a \boldsymbol{\kappa}}\left(\mathcal{S}^{-n} \boldsymbol{r}\right)=\exp (i \boldsymbol{\kappa} \cdot \boldsymbol{n}) \psi_{a \boldsymbol{\kappa}}(\boldsymbol{r}) .
$$

This implies that wave functions only in one unit cellwhatever its shape-determine the electronic structure of the whole, extended system.

You may recognize that the theorem above is nothing but Bloch's theorem, merely written with unconventional symbols. Indeed, many things remain as usual. Energy eigenstates $\psi_{a \kappa}$ automatically fulfill Eq. (5), once written in a revised version of Bloch basis,

$$
|\boldsymbol{\kappa}, \mu\rangle \equiv \varphi_{\mu}(\boldsymbol{\kappa}, \boldsymbol{r})=\frac{1}{\sqrt{N}} \sum_{\boldsymbol{n}} \exp (-i \boldsymbol{\kappa} \cdot \boldsymbol{n}) \hat{D}\left(\mathcal{S}^{n}\right) \varphi_{\mu}(\boldsymbol{r})
$$

where $\varphi_{\mu}(\boldsymbol{r})$ are local orbitals and $\sum_{n} 1=N$ is the number of unit cells. This basis gives Hamiltonian diagonal in $\boldsymbol{\kappa}$,

$$
\left\langle\boldsymbol{\kappa}, \mu|\hat{H}| \boldsymbol{\kappa}^{\prime}, \nu\right\rangle=\delta\left(\boldsymbol{\kappa}-\boldsymbol{\kappa}^{\prime}\right) \sum_{\boldsymbol{n}} \exp (-i \boldsymbol{\kappa} \cdot \boldsymbol{n}) H_{\mu \nu}(\boldsymbol{n})
$$

with

$$
H_{\mu \nu}(\boldsymbol{n})=\int \varphi_{\mu}^{*}(\boldsymbol{r}) \hat{H}\left[\hat{D}\left(\mathcal{S}^{n}\right) \varphi_{\nu}(\boldsymbol{r})\right] d^{3} r,
$$

and similarly for overlap matrix elements. The total energy expressions remain the same, we only use a set of $\boldsymbol{\kappa}$ points instead of $\boldsymbol{k}$ points (extra symmetries reduce the set) [14]. Because forces are calculated as parametric derivatives of the total energy, molecular dynamics works normally and energy is conserved; simulation cell dynamics, however, are different, and the concept of pressure needs redefinition. Finally, the theorem works with any electronic structure method, whether it is ab initio or not, whether it uses real-space grids or local orbitals (plane waves are tricky), or whether the approach is numerical or analytical.

Some things, however, do change in the revised Bloch's theorem. For bulk the periodic boundary condition is an approximation, whereas here some symmetries may form cyclic groups in reality, as in benzene. For cyclic groups the $\boldsymbol{\kappa}$-point sampling is more restricted; in the above example of an achiral carbon nanotube the translational component $\kappa_{1}$ can be freely sampled between $[-\pi, \pi]$ (because for $\mathcal{S}_{1}$ periodicity is an approximation), but the rotational component accepts only the discrete values $\kappa_{2}=$ $2 \pi m / M, m=0,1, \ldots, M-1$. Group multiplication tables for $\mathcal{S}_{i}$ that have identities like $\mathcal{S}_{i}^{l_{i}}=\mathcal{S}_{j}^{l_{j}}$ make the sampling of the components of $\boldsymbol{\kappa}$ coupled, for then we must have $l_{i} \kappa_{i}=l_{j} \kappa_{j}+2 \pi m$ ( $l_{i}, m$ integers $)$. The connection between $\boldsymbol{\kappa}$ - and $\boldsymbol{k}$ points is as follows. If $\mathcal{S}_{1}$ is a translation along $\boldsymbol{L}^{1}$, then $\mathcal{S}_{1} \psi_{\text {a }}$ should give the same phase as $\mathcal{T}\left(\boldsymbol{L}^{1}\right) \psi_{a k}$, if $\psi_{a \boldsymbol{\kappa}}$ and $\psi_{a k}$ are the same physical states. Hence $\exp \left(-i \kappa_{1}\right)=\exp \left(-i \boldsymbol{k} \cdot \boldsymbol{L}^{1}\right)$, and, in general, $\kappa_{i}=\sum_{j=1}^{3} L_{j}^{i} k_{j}$ for $i=1,2,3$.

The formalism gives surprises, too. An atom can perform work on itself. This is because the total force on an atom exerted by its own periodic images - if rotations are involved-may differ from zero. Furthermore, a force $\boldsymbol{F}_{J I}$ on atom $I$ exerted by atom $J$ may not be the counterforce to the force on atom $J$ exerted by atom $I$, that is $\boldsymbol{F}_{J I} \neq-\boldsymbol{F}_{I J}$; Newton's third law appears invalid. These unorthodoxies are not bugs; remember that we simulate the whole extended system, and an atom $I$ in the primitive unit cell is different from the atom $I$ in a different unit cell-an artifact of atom indexing. Finally, note that if $\mathcal{S}_{i}$ contains rotations, also local orbitals rotate; this is implicit in the operation $\hat{D}\left(\mathcal{S}_{i}\right) \varphi_{\mu}(\boldsymbol{r})$ in Eq. (6).

We implemented this formalism using local basis in the density-functional tight-binding software НОтвіт $[15,16]$, and tested it with many finite and extended structures. We omit the details of the implementation here, and just comment on three things. First, the standard methods of electrostatics, like Ewald summation, are invalid since flexibility is required; we chose to use multipoles as they easily lend themselves for rotations and reflections. Second, the implementation can be done so that only the mappings $\boldsymbol{r}^{\prime}=\mathcal{S}_{i} \boldsymbol{r}$ are needed to build new symmetries; this requires just a couple of lines of new code. Third, implementation has a negligible computational overhead compared to translational symmetry (see Table II). Certain manipulations take more time, but the most CPU-intensive parts remain as usual.

So far our discussion has been abstract, but what can we do with the formalism in practice? While it may seem that we require a lot of symmetries, the main point of this Letter is quite the opposite: we require fewer symmetries than before. Selected examples of usage are shown in Table I, and we believe more application areas can be discovered, once the new concepts are mastered.

Now we leave the general discussion, and give one practical example of usage: we investigate twisted armchair graphene nanoribbons (AGNRs) [20]. We choose this example for the existing literature, but also for the possibility to go beyond standard chiral symmetry.

Figure 1(a) shows a piece of an infinitely long 20AGNR, with a twist $\chi=360^{\circ} / 290.7 \AA=1.2^{\circ} / \AA$, within one conventional unit cell of 2720 atoms. The minimal unit cell, enabled by the new formalism, in turn, has 20 atoms [atoms $A$ in Fig. 1(b)], and is accompanied by two symmetry operations: $\mathcal{S}_{1}$ is $L=4.2 \AA$ translation, followed by $L \chi=5.04^{\circ}$ rotation, and $\mathcal{S}_{2}$ is $L / 2=2.1 \AA$ translation, followed by $182.52^{\circ}$ rotation $\left(=180^{\circ}+\chi L / 2\right)$. The whole system can be built from one unit cell by $\mathcal{S}_{1}^{m_{1}} \mathcal{S}_{2}^{m_{2}}$ 
TABLE I. Selected examples on the approach usage. The coordinates in parentheses mean the sense of the symmetry operation.

\begin{tabular}{|c|c|}
\hline Operations & Examples of usage and notes \\
\hline $\mathcal{S}_{1}(z)$ rotation & Bend tubes, wires, ribbons [4] \\
\hline $\mathcal{S}_{1}(z)$ rotation and $\mathcal{S}_{2}(z)$ translation & Bend membranes, slabs \\
\hline $\mathcal{S}_{1}$ joined rotation $(z)+\operatorname{translation}(z)$ & Twist nanotubes, wires, ribbons, DNA, simulate springs and coils [17-19] \\
\hline $\begin{array}{l}\mathcal{S}_{1}(x) \text { and } \mathcal{S}_{2}(y) \text { two rotations around } \\
\text { the same origin }\end{array}$ & $\begin{array}{l}\text { Simulate spherical symmetry; solid and liquid membranes, such as mono- and } \\
\text { multilayer graphene and lipid bilayers. }\left[\mathcal{S}_{1}(x) \text { and } \mathcal{S}_{2}(y) \text { commute approximately }\right. \\
\text { if rotation angles are small, and curvature can be treated as a local property.] }\end{array}$ \\
\hline $\mathcal{S}_{1}(x), \mathcal{S}_{2}(y)$ rotations around different origins & Simulating arbitrary Gaussian curvature, saddle structures (approximately) \\
\hline $\mathcal{S}_{1}$ translation $(x)+$ reflection $(y z)$ & $\begin{array}{l}\text { Structures with repeating units } \ldots A B B A \ldots \text {, using } A B \text { unit; many waves can be } \\
\text { simulated using half the wavelength }\end{array}$ \\
\hline $\begin{array}{l}\mathcal{S}_{1}(x), \mathcal{S}_{2}(y) \text { translations, } \mathcal{S}_{3}(x y) \text { reflection } \\
{[+ \text { optional translation }(x \text { or } y)]}\end{array}$ & $\begin{array}{l}\text { Computational surface science; reflection doubles the surface slab thickness with } \\
\text { half the number of atoms }\end{array}$ \\
\hline Normal point group symmetries & Finite symmetric molecules and clusters \\
\hline
\end{tabular}

with $m_{1}=0, \pm 1, \pm 2, \ldots$ and $m_{2}=0,1$. Note that $\mathcal{S}_{2}^{2}=$ $\mathcal{S}_{1}$ and hence $\kappa_{2}=\kappa_{1} / 2+\pi m_{2}$, while $\kappa_{1}$ is freely sampled. (While this was our choice for the symmetry operations, another, and equally sufficient, choice would have been to use only $\mathcal{S}_{2}$ with $m_{2}=0, \pm 1, \pm 2, \ldots$ )

Table II shows the wall-clock times for selected simulations. The simulations with $\chi=0$ show that the new formalism has no computational overhead compared to translation. The simulations with $\chi=1.2^{\circ} / \AA$ show that finite twist affects simulation times with neither minimal

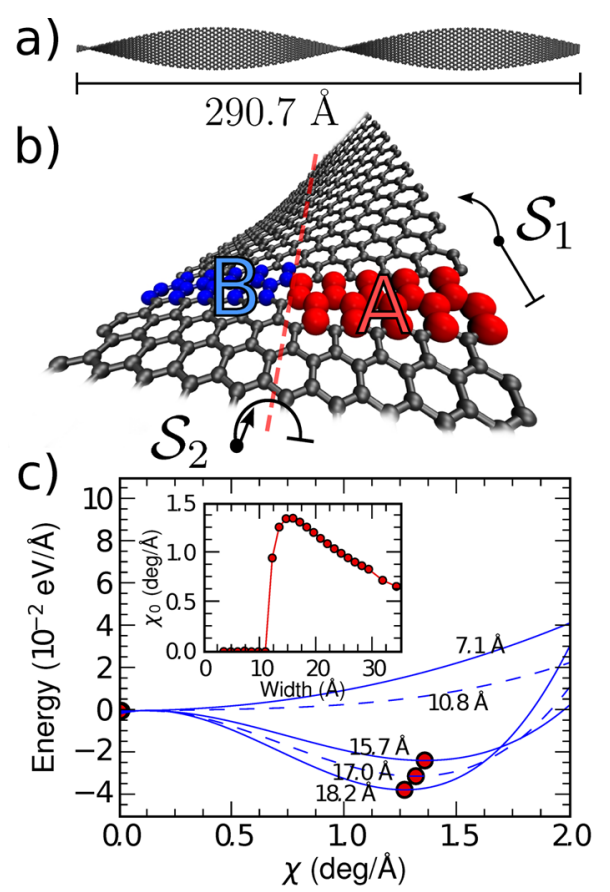

FIG. 1 (color online). (a) Conventional unit cell of 20-AGNR with a twist $\chi=1.2^{\circ} / \AA$ (2720 atoms). (b) Minimal unit cell (atoms $A$ ), with illustrations of $\mathcal{S}_{1}$ and $\mathcal{S}_{2} . \mathcal{S}_{1}$ is the chiral operation and $\mathcal{S}_{2}$ transforms $A \rightarrow B$. (c) Energies for AGNRs as a function of $\chi$ for selected ribbon widths. Minimum of the curve gives the spontaneous twist $\chi_{0}$. We used a $20 \times 2 \boldsymbol{\kappa}$-point mesh. Inset: $\chi_{0}$ as a function of AGNR width. nor chiral cells. The translational cell was too large for direct simulation, and the timing was estimated from the

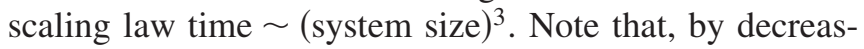
ing $\chi$, the translational cell size-along with its timingcould easily be grown indefinitely. The point here is that twists even smaller than $1.2^{\circ} / \AA$ will be required to investigate the relevant physics of a 20-AGNR. Conventional quantum-mechanical simulation is practically impossible. We also remark that, given compatible $\boldsymbol{\kappa}$-point samplings, energies and forces from different types of cells are the same within floating-point precision.

Figure 1(c) shows AGNRs' energies as a function of twist. For ribbons wider than $\sim 12 \AA$ the energy is at minimum with nonzero $\chi=\chi_{0}$; ribbons twist spontaneously. This confirms earlier predictions by classical potentials (using thousands of atoms) [5,9] and finite element modeling [3]. The physical reason for twisting is the compressive edge stress that elongates edges with respect to ribbon's center $[5,9,21,22]$. The stress we get

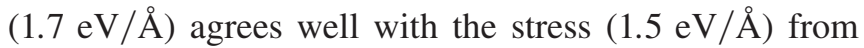
previous density-functional calculations [9]. For wide ribbons we get scaling $\chi_{0} \sim 22^{\circ} /$ width, and the difference to classical scaling $\chi_{0} \sim 18^{\circ} /$ width of Ref. [5] comes mainly from quantum mechanics: the edge stress resides not only at the edge, but extends more into ribbon's center-a feature hard to reproduce by classical potentials [9]. This

TABLE II. Timings for 20-AGNR with different twists. Time is the wall-clock time per molecular dynamics step, calculated with a standard present-day desktop computer.

\begin{tabular}{lccc}
\hline \hline \multicolumn{1}{c}{ Unit cell } & Atoms & $\chi(\mathrm{deg} / \AA)$ & Time (s) \\
\hline Minimal [like $A$ in Fig. 1(b)] & 20 & 0 & 1.51 \\
Chiral [like $A+B$ in Fig. 1(b)] & 40 & 0 & 2.75 \\
Translational (like chiral) & 40 & 0 & 2.75 \\
Minimal [A in Fig. 1(b)] & 20 & 1.2 & 1.51 \\
Chiral [ $A+B$ in Fig. 1(b)] & 40 & 1.2 & 2.75 \\
Translational [Fig. 1(a)] & 2720 & 1.2 & $9.26 \times 10^{4}$ \\
\hline \hline
\end{tabular}

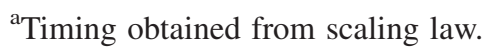



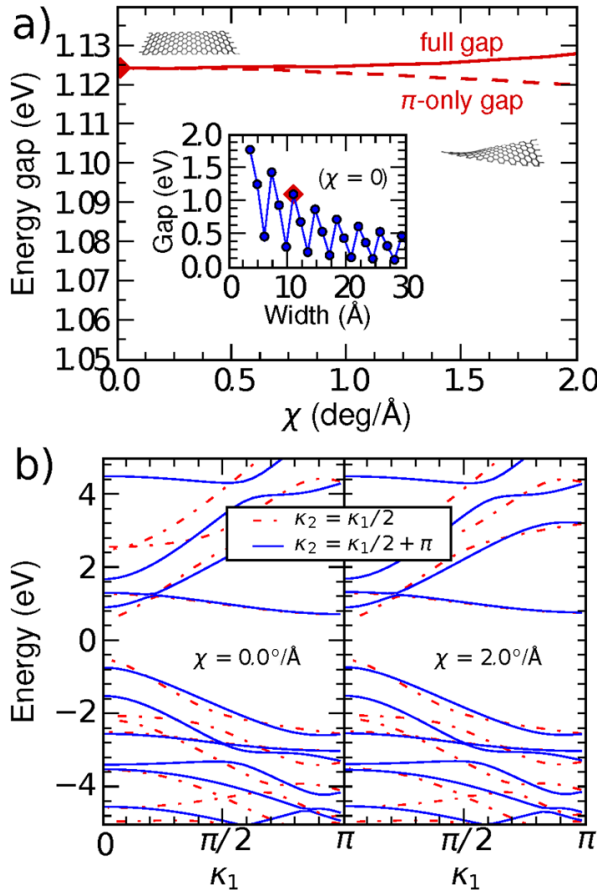

FIG. 2 (color online). (a) Energy gap of a $10.8 \AA$ wide $10-$ AGNR as a function of $\chi$. The $\pi$-only gap is obtained by treating the unhybridized $2 p_{z}$ electrons as $s$ electrons. Inset: energy gaps as a function of AGNR width for $\chi=0$. (b) Band structures for flat and twisted ribbons, plotted as a function of $\kappa_{1}$. The symmetry operation $\mathcal{S}_{2}$ brings another dimension $\kappa_{2}$ to band structure plots. Calculation does not include spin.

is also why we have no spontaneous twist for narrow ribbons. Ultimately, very wide ribbons should show bifurcation into flat ribbons with ripples at the edges, but we will not discuss that here [3,5].

As argued in the abstract, the electronic properties ought to be investigated together with mechanical properties; this requires quantum mechanics. It is known that AGNRs have a gap due to the confinement of the finite width, and our gaps [inset in Fig. 2(a)] agree well with density-functional calculations of Ref. [20]. But what happens to electronic structure when ribbons get twisted? Figure 2(a) shows that twisting changes 10-AGNR's gap very little-this is generic for all AGNRs. The gap from $\pi$ electrons alone shows further that $s p$ rehybridization is negligible. Even the band structures of flat and twisted ribbons [Fig. 2(b)] are nearly identical. This suggests that, contrary to CNTs [23], the electronic properties of GNRs are remarkably robust against twisting.

To conclude, we hope to have illustrated how modest revision of Bloch's theorem enables versatile material distortions with quantum mechanics included. However, excess emphasis on quantum mechanics causes undue discrimination of classical methods-the formalism works equally with classical force fields, finite element methods, or coarse-grained simulations, and equally when applied to, say, liquid-phase cell membranes, fluid flow through bent pipes, or electron transport.

We acknowledge the Academy of Finland for funding, H. Häkkinen for support, and the Finnish IT Center for Science (CSC) for computational resources.

Note added.-Recently we became aware of similar earlier work by Dumitrică et al. [24], cited here for the readers' benefit.

*Corresponding author.

†pekka.koskinen@iki.fi

[1] F. Bloch, Z. Phys. 52, 555 (1929).

[2] A. H. Castro Neto, Mater. Today 13, 12 (2010).

[3] V. B. Shenoy, C.D. Reddy, A. Ramasubramaniam, and Y. W. Zhang, Phys. Rev. Lett. 101, 245501 (2008).

[4] S. Malola, H. Häkkinen, and P. Koskinen, Phys. Rev. B 78, 153409 (2008).

[5] K. V. Bets and B. I. Yakobson, Nano Res. 2, 161 (2009).

[6] J.C. Meyer, A. K. Geim, M.I. Katsnelson, K. S. Novoselov, T.J. Booth, and S. Roth, Nature (London) 446, 60 (2007).

[7] W. Bao, F. Miao, Z. Chen, H. Zhang, W. Jang, C. Dames, and C. N. Lau, Nature Nanotech. 4, 562 (2009).

[8] L.D. Landau and E. M. Lifshitz, Theory Of Elasticity (Pergamon press, Oxford, 1970).

[9] B. Huang, M. Liu, N. Su, J. Wu, W. Duan, B. L. Gu, and F. Liu, Phys. Rev. Lett. 102, 166404 (2009).

[10] K. N. Kudin, G. E. Scuseria, and B. I. Yakobson, Phys. Rev. B 64, 235406 (2001).

[11] C. T. White, D. H. Robertson, and J. W. Mintmire, Phys. Rev. B 47, 5485 (1993).

[12] V. N. Popov, New J. Phys. 6, 17 (2004).

[13] D.-B. Zhang and T. Dumitrică, Appl. Phys. Lett. 93, 031919 (2008).

[14] R. M. Martin, Electronic Structure: Basic Theory and Practical Methods (Cambridge University Press, Cambridge, U.K., 2004).

[15] P. Koskinen and V. Mäkinen, Comput. Mater. Sci. 47, 237 (2009).

[16] Hotbit wiki https://trac.cc.jyu.fi/projects/hotbit.

[17] H. M. Lawler, J. W. Mintmire, and C. T. White, Phys. Rev. B 74, 125415 (2006).

[18] L. Pan, M. Zhang, and Y. Nakayama, J. Appl. Phys. 91, 10058 (2002).

[19] H. Ko, Y. Pikus, C. Jiang, A. Jauss, O. Hollricher, and V. V. Tsukruk, Appl. Phys. Lett. 85, 2598 (2004).

[20] Y.-W. Son, M. L. Cohen, and S. G. Louie, Phys. Rev. Lett. 97, 216803 (2006).

[21] S. Jun, Phys. Rev. B 78, 073405 (2008).

[22] C. D. Reddy, A. Ramasubramaniam, V. B. Shenoy, and W.-W. Zhang, Appl. Phys. Lett. 94, 101904 (2009).

[23] L. Yang and J. Han, Phys. Rev. Lett. 85, 154 (2000).

[24] T. Dumitrică and R. D. James, J. Mech. Phys. Solids 55, 2206 (2007); D.-B. Zhang, M. Hua, and T. Dumitrică, J. Chem. Phys. 128, 084104 (2008); D.-B. Zhang, R. D. James, and T. Dumitrică, Phys. Rev. B 80, 115418 (2009). 